\title{
INTERPLAY BETWEEN ART AND SCIENCE IN EDUCATION: "MUSIC" BASED APPROACH IN NANOSCIENCES
}

\author{
Samo Kralj, Boris Aberšek \\ University of Maribor, Slovenia \\ E-mail: samo.kralj@uni-mb.si, boris.abersek@uni-mb.si \\ Irena Kralj \\ High School in Celje, Slovenia \\ E-mail: nenikralj@gmail.com
}

\begin{abstract}
Music can be viewed as a structure formed by notes. Different structures in music have potential to yield enormously rich diversity of different melodies. Music is a typical example where a structure defines a property. Similar concepts could be also exploited in education, in presented case in nano-sciences, which are typical representatives of soft materials the structure of which can be strongly manipulated with local geometry and presence of appropriate nanoparticles. The objects of study, named also LC shells, will be exploited as basic unit elements for future soft colloidal crystals. A different arrangement of colloids within the crystal would result in different physical properties in a similar way as different packing of atoms results in different crystals made of real atoms.

In presented research will be demonstrated, how relevant basic mechanisms in thin films of nematic liquid crystals could be explained in a classroom and used as a case study, also for explanation of many other physical properties. This research topic is still in its infancy. At this stage only various defect structures in relatively simple geometries (spherical and elliptical) will be analyzed. There is a need to find simple ways to control sensitively the valence of LC shells and in particular to develop strategies to assemble them in crystal structures of desired symmetry. This would allow tailoring specific optical dispersion relations or other physical property of interest and make new ways to teach different physical properties on the "music « based approach.
\end{abstract}

Key words: teaching/learning strategies, »music« based approach, topological defects, localized states, nanoscience, soft materials, liquid crystalline shells.

\section{Introduction}

History manifests strong interplay between art and science. Roughly 2.500 years ago Pitagora was the first to observe connection between mathematics and music. Later Johann Sebastian Bach discovered universality in music, which is nowadays broadly applied in science. Furthermore, in his several compositions he combined fine balance between "order" and "disorder" which enabled him to obtain rich diversity in music expressions. Note that the key ingredient of modern complexity theory, which explains richness of natural phenomena because the Nature is at the "edge of chaos", separating regions exhibiting chaotic behaviour and order. Cubism in art, in particular Georges Braque, inspired pioneering research in Quantum Mechanics to introduce the duality concept of Nature, where either wave or particle- type behaviour is observed. At the beginning of $20^{\text {th }}$ century Henri Poincare introduced phase space in order to solve differential equations graphically. His mathematical three-body behaviour analysis trig- 
Samo KRALJ, Boris ABERŠEK, Irena KRALJ. Interplay between Art and Science in Education: "Music" Based Approach in Nanosciences

OF EDUCA

IN THE $21^{\text {st }}$ CENTURY

Volume 61,2014

68 kson Pollock represent first artistic manifestations of fractals before they were discovered in science. After Chaos was accepted as an important branch in science, different fractal textures become ubiquitous and fashionable ingredient in paintings. Later Gyorgy Ligeti introduced fractals, representing the key signature of a chaotic behaviour, into his music compositions... These few key examples evidently show mutual connection between science and art, where experiences gained in one branch could be beneficial to the other branch.

Physical science, particular nanoscience could also benefit from music. Namely, various melodies are in fact different structures composed of finite number of discrete units - notes. By changing their relative position, duration, dynamics (i.e. rhythm) different melodies and music styles emerge. This paradigm, where different structures define qualitatively different global behaviour, can be applied in nanoscience. Currently, main focus in constructing nano-based new materials is based on developing new nano-units. However, music suggests that different "properties« are not always directly defined by the basic building blocks themselves but by the way they are organized into hierarchies. Music is in fact a hierarchical system, where specific patterns are nested within larger patterns. Similar mechanisms might be applied in designing new nano-composites with exotic properties.

As an illustration of »music« based approach we present different structures in thin liquid crystalline (LC) films (De Gennes, Prost, 1993) and LC shells (Vitelli, Nelson, 2006). The latter represent thin films of nematic LCs covering a colloidal particle. As "units" we consider topological defects (TDs) in LC orientational ordering (Mermin, 1976). Our research is theoretical and we use two dimensional Landau-type approach in terms of complex order parameter (De Gennes, Prost, 1993, Kralj, et al., 2011). Using it we express the free energy density in terms of invariants formed by the Taylor expansion in $Q$ and its first spatial derivatives. Different metastable or stable structures of orienational ordering defined by $Q$ are obtained numerically by solving corresponding Euler-Lagrange equations (Kralj, et al., 2011). Different patterns of TDs by exploiting their coupling with the curvature of LC films or shells and TDs are imposed and shown how such systems could be in future exploited to form the so called scaled crystals (Nelson, 2002), where microscopic LC-shells are analogues of atoms in conventional crystals.

\section{Localised States in Continuum Theories}

In nature localised "objects" with well defined fixed shapes (i.e., constant in time) reminding us of notes in music have been observed and referred as particles. At a fundamental level, in physics ordering of a system is commonly described in terms of continuum fields. Scientists have for years searched for nonlinear field-type theories yielding stable localised states, reminding us of particles. Skyrme was the first to describe nuclear particles as localised states (Skyrme, 1961), so called "Skyrmions". In the following years they discovered Skyrmions in field-type theories describing variety of completely different physical systems, where characteristic lengths varied from microscopic (Al'Khawaja, Stoof, 2001) to cosmological scales (Durrer, et al., 2002). In these pioneering studies Skyrmions have been found only under non-equilibrium conditions or have been stabilised by external fields. However, latter stable configurations were discovered, for example in magnetic systems (Rößler1, et al., 2006), where Skyrmions are stabilised by chirality. Chirality refers to symmetry breaking between left and right handedness. Therefore, a chiral system is characterised by a preferred handedness and consequently the system lacks the inversion symmetry.

In a wider context Skyrmions are members of the family of topological defects (TDs) (Mermin, 1976). Namely, their local stability is due to topological reasons. Consequently, their main property is defined by a topological quantity, so called topological charge, which is independent of microscopic details of a system. TDs appear at least temporary in systems which are reached via a symmetry breaking phase transition. In fact, last majority of structures in condensed matter and even structure of the universe were reached via such transitions. For this 
reason TDs are ubiquitous in nature, and science of topological defects is universal. For example, the first theory of coarsening dynamics of topological defects was developed in cosmology (Zurek, 1976). However, in most commercial applications based on TDs liquid crystals are used as working materials (De Gennes, Prost, 1993).

\section{Topological Defects in Liquid Crystals}

Skyrmion-type stable configurations are observed also in liquid crystals (LCs) (De Gennes, Prost, 1993). Most famous are Blue phases (BPs) (Cordoyiannis, et al., 2013) and Twist Grain Boundary (TGB) (Wright, Mermin, 1989) phases which are dominated by stable lattices of such topological defects. These are in BPs and TGBs commonly referred to as line disclinations and screw dislocations, respectively. Note that the discovery of the latter structure was guided by mathematical similarity in describing translational ordering in LCs and superconductivity in superconductors (Chaikin, Lubensky, 1995). Namely, in both systems the primary order parameter field is a complex quantityy $=h e^{i f}$, where the modulus $h$ (degree of ordering) and the phase factor $f$ are real numbers. In this analogy i) TGB and the ii) screw dislocation in LCs are analogues of the i) Abrikosov phase and the ii) magnetic vortex in superconductors.

Liquid crystals (De Gennes, Prost, 1993) are typical soft matter representatives, displaying ordering between conventional liquids and solid crystals. The defining property of soft materials is easiness of their response to even modest external perturbations. Therefore, LCs can be relatively easily manipulated by confining surfaces and/or external electric or magnetic fields. LCs are optically transparent and anisotropic and consequently their structural characteristics could be relatively easily monitored using, for example, optical polarization microscopy. Furthermore, richness of different LC phases and structures enables existence of any topological defects encountered in Nature (Mermin, 1976). Consequently, LCs are most convenient testing ground systems to study fundamental properties of topological defects.

The nematic phase is the simplest LC phase (De Gennes, Prost, 1993). For presentation purposes we henceforth restrict to thermotropic LCs in which the nematic phase is reached from the isotropic (ordinary liquid) phase by lowering temperature. We also set that on average LC molecules behave as rod-like objects with head-to-tail symmetry (i.e., up and down positions are equivalent). In bulk samples (relatively large sample where we neglect boundaries) in the nematic phase LC molecules are on average aligned along a single (the so called symmetry breaking) direction. The nematic local average orientational ordering at a site $\vec{r}$ is commonly described by the unit vector $\vec{n}(\vec{r})$, called the nematic director field (De Gennes, Prost, 1993). The degree of ordering about this direction is quantified by the scalar nematic order parameter field I ( $\vec{r}$ ) (De Gennes, Prost, 1993). For rigidly aligned LC molecules it has maximal value. On the other hand $\mathrm{I}=0$ reflects absence of orientational ordering, where all orientations are equivalent.

In a nematic LC phase topological defects can be relatively easily visualized. Some simple examples are sketched in Figure 1. They refer to points or lines, where the director field is not uniquely defined. In most cases a large number of TDs appear temporary in relatively fast LC structural or phase changes enforcing local frustrations, which could not be relieved by continuous spatial variations in $\vec{n}(\vec{r})$. Note that local free energy costs of introducing a topological defect are in general relatively large. Therefore, in most cases systems tend to avoid presence of TDs. However, due to topological reasons one could not get rid of an isolated TD if the director field on any surface enclosing the defect is fixed. One can decrease number of TDs only if a pair of defects of opposite topological charge annihilate each other (Kralj, et al., 2011).

Principal property of a topological defect is its topological charge $m$ (Mermin, 1976), which is a topological invariant. Therefore, it does not change if we make continuous changes within a volume containing the defect if conditions at the volume's boundaries are unaltered. In 
Samo KRALJ, Boris ABERŠEK, Irena KRALJ. Interplay between Art and Science in Education: "Music" Based Approach in Nanosciences

OF EDUCATION

IN THE $21^{\text {st }}$ CENTURY

Volume 61,2014

70 our study we will restrict to two dimensional systems (2D). In 2D the topological charge is equivalent to the winding number (also referred to as the Frank index (De Gennes, Prost, 1993)), which can be simply visualized. The winding number is determined by the orientational field surrounding TD. It counts total angular change in orientation of $\vec{n}$ on encircling TD clock-wise using an arbitrary path divided by $2 \partial$. If TD exists then $m$ equals to half integer or integer. TDs exhibiting $m>0$ and $m<0$ are commonly referred to as defects and antidefects, respectively. In Figure 1 are depicted schematic plots of simple TD structures bearing charges $m=1 / 2$ , $m=-1 / 2, m=1$ and $m=-1$. Note that TDs in most cases behave in a similar way as electrically charged particles or fundamental particles in particle physics bearing some quantized charge in general (Rößler1, et al., 2006). Namely, the total charge of a closed system is a conserved quantity. TDs of opposite (same) charges attract (repel) each other and can annihilate into a defectless state.

a)

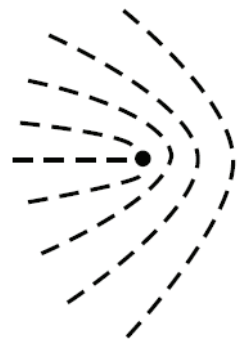

b)

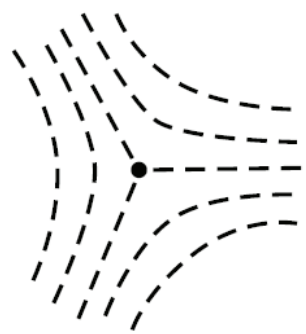

c)

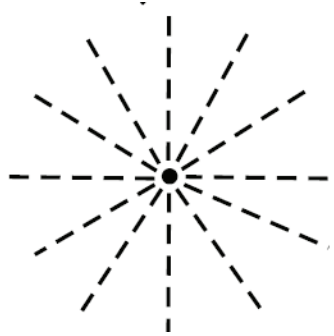

d)

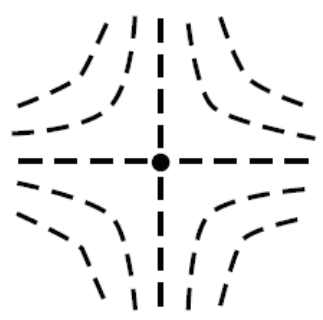

Figure 1: Topological defects in orientational ordering are presented by topological charge which is in two dimensional systems equivalent to the winding number $m$. a) $m=1 / 2$, b) $m=-1 / 2, c) m=1$, d) $m=-1$.

\section{Soft Nano-Composites: "Music" Based Approach}

Recent years face increased interest into soft nano-composites (Nelson, 2002, Cordoyiannis, et al., 2013) and also on impact of nanoparticles (NPs) on biological membranes (Vitelli, Turner, 2004). Soft nano-composites typically consist of a soft matrix containing NPs. In such a way unique properties of soft materials and nanoparticles are combined. It is of interest that the resulting material either exhibits anomalously enhanced quantitative properties (with respect to "pure samples" in absence of nanoparticles) or qualitatively new properties.

In particular it has been shown, that TDs within soft matrices could strongly interact with nanoparticles (Cordoyiannis, et al., 2013). Several studies reveal that appropriately surface decorated NPs could be efficiently trapped within cores of topological defects in various LC phases. Furthermore, appropriate NPs could substantially increase (by orders of magnitude) the stability range of relevant defect lattices due to the universal Defect Core Replacement (DCR) mechanism (Cordoyiannis, et al., 2013). This describes reduction of condensation free energy penalty related to existence of defect's core by partially replacing its costly core by volume of NPs. This mechanism is efficient if NPs do not significantly disrupt a local LC environment. In such a way one could control positioning of NPs via manipulation of positions of TDs. The latter could be relatively easily manipulated in different ways due to the soft character of LC matrix. Different responses are obtained if NPs have substantial impact on LC orientational ordering. For example, let us assume that a spherical NP enforces radial distribution of $\vec{n}$ at the NP-LC interface. Therefore, it locally acts as a topological defect represented by $\mathrm{m}=1$, see Figure 1c. If the total topological charge of the system equals zero (this is the case if far from NP liquid crystalline ordering is preferently homogeneously aligned along a single direction), then LC matrix must respond by creating antidefects (for example one with $\mathrm{m}=-1$ or two with $\mathrm{m}=-1 / 2$ ) to compensate the effective charge introduced by the nanoparticle. 
Furthermore, in 2D systems it has been demonstrated that the local surface curvature can have strong impact on position and even number of TDs (Kralj, et al., 2011, Vitelli, Turner, 2004). In particular, the Gaussian curvature $G$ plays a significant role. It can be expressed with local principal radia $R_{1}$ and $R_{2}$ of curvature as $G=1 /\left(R_{1} R_{2}\right)$. It has been shown that $G$ has similar impact on topological defects as electrical field on electrical charges. Namely, regions with positive (negative) value of $G$ attract TDs with negative (positive) charge $m$. Furthermore, surfaces exhibiting regions with $G<0$ and $G>0$ have potential to generate pairs \{defect, antidefect\} (Vitelli, Nelson, 2006, Vitelli, Turner, 2004, Jesenek, et al., 2013). Therefore, interactions between TDs and NPs within a LC on curved surfaces (e.g. in LC films or within biological membranes possessing) can give rise to diverse complex structures.

One way to obtain different properties of soft-matrices is to follow examples in music. In the latter case on uses few different music notes and their structural organization defines a melody. In the case of soft-matrices role of notes can be played by topological charges. They can be placed on different positions by playing with surface local curvature and/or appropriate nanoparticles. In the following we use a simple model to illustrate key mechanisms via which positioning of "notes" in orientationally ordered soft materials can be controlled.

\section{Minimal Model}

A minimal mesoscopic theory describing nematic LC orientational ordering in 2D curved surface embedded in 3D coordinate system have been used. Points on a surface are parametrized by parameters $(u, v)$. Details are given in (Kralj, et al., 2011). In reality such LC configurations could be encountered in thin LC films, biological membranes exhibiting in-plane orientational order or other analogous systems.

We set that a local surface orientation is determined by its outer unit normal $\vec{v}$. A local average orientation of a LC rod-like molecule is described by $\vec{n}$. The corresponding tensor order parameter is defined as

$$
\underline{Q}=\mathrm{I}\left(\vec{n} \otimes \vec{n}-\vec{n}_{\perp} \otimes \vec{n}_{\perp}\right)
$$

where $\underline{I}$ stands for the Identity tensor, $\vec{n} \times \vec{n}_{\perp}=\vec{v}$, and the scalar order parameter I measures the degree of ordering.

The corresponding orientational free energy density $f$ can be expressed as a Landau-type expansion in terms of $Q$ taking into account symmetry allowed terms. In the lowest order approximation it is given by (Kralj, et al., 2011)

$$
f=-A \operatorname{Tr} \underline{Q}^{2}+B(\operatorname{Tr} \underline{Q})^{2}+k\left|\nabla_{s} \underline{Q}\right|^{2} .
$$

Here $A, B, k$ are positive material constants and $\square_{\mathrm{s}}$ marks the surface gradient operator.

In elastically undistorted bulk system the equilibrium degree of ordering in orientationally ordered phase is given byl ${ }_{c}=\sqrt{A /(2 B)}$. The competition between the condensation (the first two terms in Eq.(2)) and the elastic (the last term in Eq.(2)) free energy contributions defines the order parameter correlation length of the system, which is roughly given by $\mathrm{x} \sim \sqrt{k / A}$. Equilibrium LC configurations are obtained via numerical mimization of the free energy for a given 2D geometry (Kralj, et al., 2011).

\section{Results of Theoretical Case Study}

In the following we demonstrate strong impact of curvature and also simple NPs on position and number of topological defects of charge $m= \pm 1 / 2$ which play in our analogy the role of "notes". We refer to $m=1 / 2$ and $m=-1 / 2$ TDs as defects and antidefects, respectively. For demonstration reason we use as simple as possible model capable to display variety of different 
Samo KRALJ, Boris ABERŠEK, Irena KRALJ. Interplay between Art and Science in Education: "Music" Based Approach in Nanosciences

OF EDUCATIOI

IN THE $21^{\text {st }}$ CENTURY Volume 61, 2014

structures which have potential to strongly influence resulting physical properties. For this purpose we consider thin nematic films covering objects exhibiting spherical topology. Furthermore, we restrict to axially symmetric shapes. For better visualization we also consider geometries where typical linear dimension $R$ of a shape is larger but comparable to $\mathrm{x} \sim \sqrt{k / A}$. The latter length roughly determines the size of the core of defect, where degree of ordering is locally strongly distorted. Note that the core of a defect roughly corresponds to the "body shape" of "notes". Namely, in most situations the core structures (at least degree of ordering) are relatively weakly influenced by details of surrounding LC ordering (Kralj, Virga, 2001).

In Figure $2 \mathrm{a}$ we show positioning of TDs on an ideal sphere. Note that due to topological reasons such geometry enforces total topological charge equal to $m_{\text {tot }}=2$. Exactly at a defect origin the nematic ordering is melted (i.e., I =0), making them clearly visible in our simulations. Relative position of defects and their number and core structure is better visualized by plotting degree of ordering in the $(u, v)$ plane in Figure $2 \mathrm{~b}$ and Figure $2 \mathrm{c}$. In the former we plot $\mathrm{I}(u, v)$ and in the latter the director field. Here $u \in[0,2 \mathrm{p}[$ and $v \in[0, \mathrm{p}[$ describe the azimuthal and the zenithal angle of the spherical coordinate system. Figure 2c clearly reveals that four TDs bearing $m=1 / 2$ are formed. Note that in this case the Gaussian curvature is constant over the sphere. For this reason position of TDs is dominated by mutual repulsion among defects. Consequently, TDs occupy vertices of tetrahedral in order to maximize their mutual separation (Vitelli, Nelson, 2006).

(a)

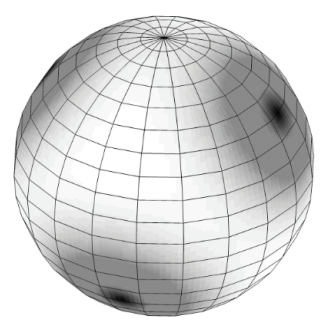

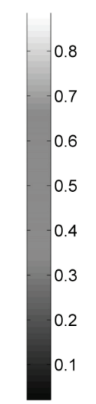

(b)

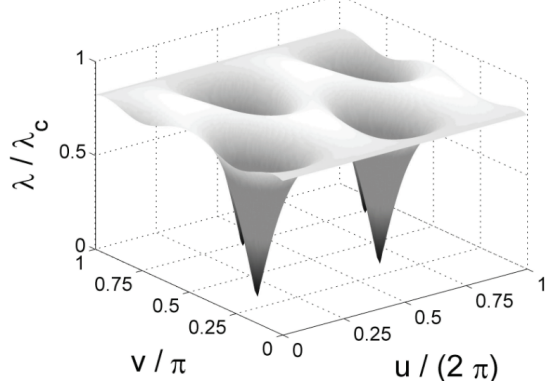

(c)

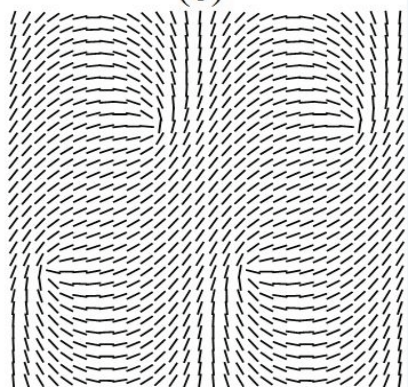

Figure 2: Topological defects in orientational order within a thin LC film covering the surface of a sphere. a) Degree of ordering superimposed on the sphere. Defects are localized at regions exhibiting black color. The bar at the

right hand determines the value $/ / I_{c}$. b) Two dimensional variation of I / I in the $(u, v)$ plane, where $u$ and $v$ describe the azimuthal and zenithal angle of the spherical coordinate system. c) The corresponding director field distribution in the $(u, v)$ plane. 
In Figures 3 we demonstrate impact of curvature on positions of defects. For this reason we consider prolate and oblate ellipsoids. In these cases positive curvature exhibits maximum at the poles and equatorial ring, respectively. With increasing prolateness pairs of defects are approaching poles (Figure 3a). On the contrary, increasing oblateness the defects are approaching the equatorial line (Figure $3 \mathrm{~b}$ ). Note that if we enforce two spherical NPs at the poles of a sphere which impose radial director field at their boundaries director field would be aligned along meridians. In this case TDs are absent. Namely, such NPs effectively act as TDs each bearing topological charge $m=1$, imposing the total topological charge $m_{\text {tot }}=2$ to the system. Consequently, in this case "real" TDs are absent.

Structures shown in Figures 4 exhibit spatially dependent Gaussian curvature and possess both regions with $G>0$ and $G<0$. Negative value of $G$ is established at the necks of structures. In Figures a) to d) we progressively increase the average mean curvature of structures. In Figure $4 \mathrm{a}$ and $4 \mathrm{~b}$ there are four defects as in the spherical case (Figure 2a). However, due to spatially nonhomogeneous dependence of $G$ the defects are pushed towards the poles of objects. For strong enough local curvatures new pairs defect-antidefect can appear. In Figure 4c we show the configuration bearing eight TDs. Three defects are positioned at each bead and at each neck one antidefect resides. In Figure 4d we have 12 TDs.

a)

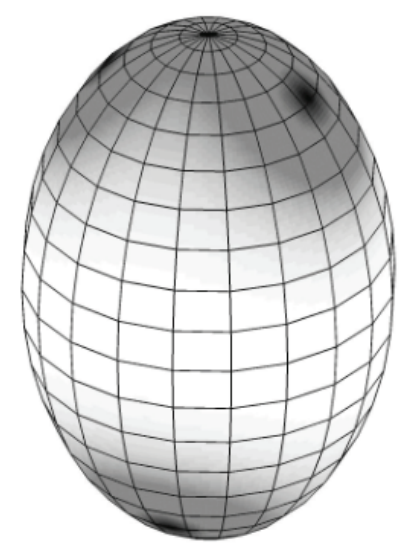

c)

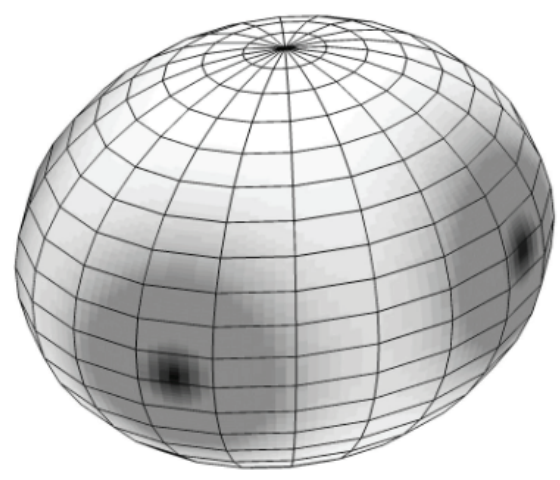

PROBLEMS

OF EDUCATION

IN THE $21^{\text {st }}$ CENTURY

Volume 61,2014

73 
LEMS

OF EDUCATION

IN THE $21^{\text {st }}$ CENTURY

Volume 61,2014 a)

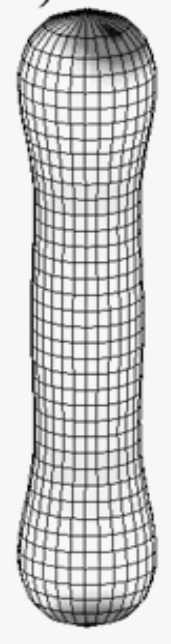

b)

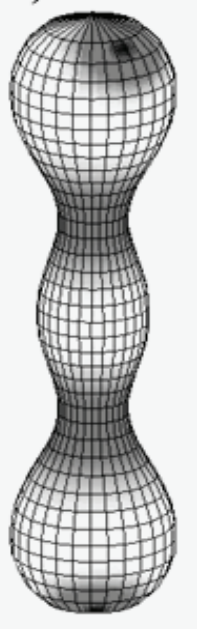

c)

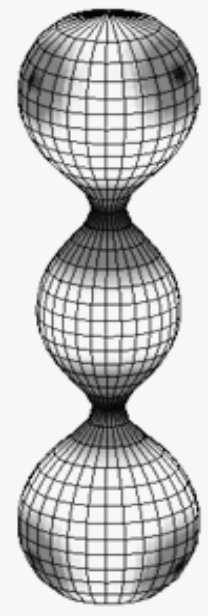

d)

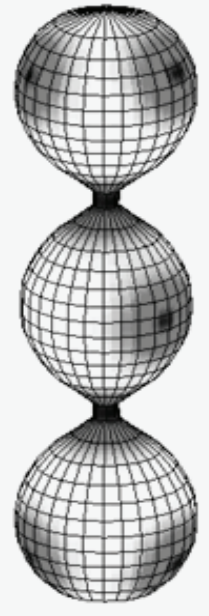

Figure 4: Shapes exhibiting spherical topology enforcing $\boldsymbol{m}_{\text {tot }}=2$. Average curvature of structures gradually increases from a) to d). Configurations in a) and b) possess 4 defects with $m=1 / 2$. Within structures in c) and d) curvature is strong enough to generate additional pairs (defect, antidefect).

\section{Summing-up}

Music is composed of a finite set of different music notes. Importantly, by varying structure formed by these notes qualitatively different music styles could be formed. Music is a typical example where a structure defines a property. This concept could be followed in education when nano-sciences are the subyect of lecture. In presented contribution relevant basic mechanisms in nematic liquid crystals, which are typical representatives of soft materials had been presented.

If thin 2D nematic LC films have been considered the degree of orientational ordering with the tensor order parameter could be described. For demonstrative purpose could be used the spherical topology, which by itself enforces the total topological charge $m_{\text {tot }}=2$, which showed that by manipulating a local geometry (i.e. the Gaussian curvature $G$ ) a number and position of topological defects bearing topological charge $m= \pm 1 / 2$ could be controlled. Here TDs in LCs mimic notes in music. Research demonstrated that TDs can be efficiently targeted to different positions within a LC matrix by playing with local curvature. In particular defects (TDs characterized by $m=+1 / 2$ ) are attracted to regions exhibiting the maximal positive value of $G$. On the contrary antidefects (TDs characterized by $m=-1 / 2$ ) tend to be assembled at regions exhibiting minimal negative value of $G$. Furthermore, by imposing regions exhibiting both positive and negative values of $G$ arbitrary number of additional TDs could be introduced provided that a LC region is large enough. Positions and number of TDs could be in addition manipulated by appropriate NPs. For example, if a nanoparticle imposes a radial-type director profile in its close neighborhood it effectively affects surrounding LC medium like a topological defect bearing topological charge $m=1$. First of all, such NP can strongly influence positions of neighboring TDs. Next, due to the topological charge conservation these NPs can change number of TDs. Therefore, by exploiting geometry and NPs different spatial patterns of "notes" (i.e. TDs) can be formed. Different patterns could in general enable different physical responses. In LCs, which are famous for their tunability and responsiveness to local stimuli, one could 
realize rich variety of patterns of TDs exhibiting qualitatively different symmetries. Note that several examples in nature demonstrate that symmetry strongly influences physical properties. For example, diamond, carbon nanotubes, graphene and graphite are all composed of Carbon atoms and they only differ only in its structural spatial (i.e. symmetry) arrangement.

As a final Remarque, objects of our study, named also LC shells (Vitelli, Nelson, 2006, (Kralj, et al., 2011, Nelson, 2002), could be exploited as basic unit elements for future soft colloidal crystals. Here different arrangement of colloids within the crystal would result in different physical properties in a similar way as different packing of atoms results in different crystals made of real atoms. Namely, Nelson (Nelson, 2002) proposed to disperse appropriately coated LC shells within isotropic media including appropriate nano-sized binders. The latter would anchor to defect sites and in this manner organize shells into regular crystal superstructures. This research topic is still in its infancy. At this stage only various defect structures in relatively simple geometries (spherical and elliptical) have been analyzed. There is a need to find simple ways to control sensitively the valence of LC shells and in particular to develop strategies to assemble them in crystal structures of desired symmetry. This would allow tailoring specific optical dispersion relations or other physical property of interest and make possibilities to explain students very complicated structures in a user friendly way.

\section{Acknowledgments}

The authors acknowledge the support of the Ministry of Education, Science and Sport of Republic of Slovenia and European Social Fund in the frame of the Project: "Innovative pedagogy 1: 1 in the light of competences of the 21st century" on Faculty of Natural Sciences of University of Maribor.
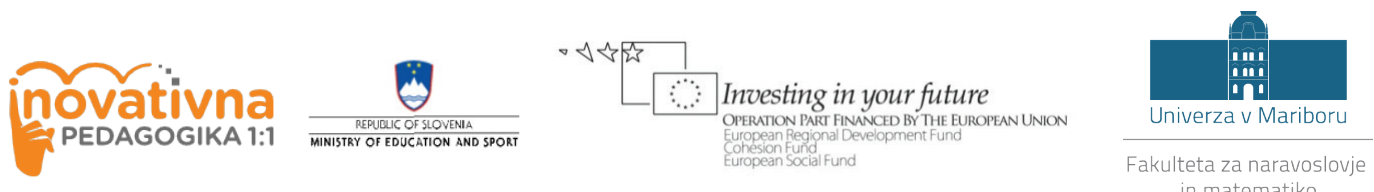

\section{References}

Al'Khawaja, U., Stoof, H. T. C. (2001). Skyrmions in a ferromagnetic Bose-Einstein condensate. Nature, 411, 918-920.

Bradač, Z., Kralj, S., Svetec, M., Žumer, S. (2003). Annihilation of nematic point defects: postcollision scenarios, Physics Review E, 67, 050702(R), 1-4.

Chaikin, P. M., Lubensky, T. C. (1995). Principles of condensed matter physics Ch. 2. Cambridge University Press, Cambridge, UK.

Cordoyiannis, G. , Jampani, V. S. R., Kralj, S., Dhara, S., Tzitzios, V., Basina, G., Nounesis, G., Kutnjak, Z., Tripathi, C. S. P., Losada-Perez, P., Jesenek, D., Glorieux, C., Musevic, I., Zidansek, A., Amenitsch, H. (2013). Different modulated structures of topological defects stabilized by adaptive targeting nanoparticles. Soft Matter, 3956-3964.

De Gennes, P. G., Prost, J. (1993). The physics of liquid crystals. Oxford University Press, Oxford.

Durrer, R., Kunz, M., Melchiorri, A. (2002). Cosmic structure formation with topological defects. Physics Report, 364, 1-81.

Jesenek, D., Perutkova, S., Gozdz, W., Kralj-Iglic, V., Iglic, A., Kralj, S. (2013).Vesiculation of biological membrane driven by curvature induced frustrations in membrane orientational ordering. International Journal of Nanomedicine, 8, 677-687.

Kralj, S., Virga, E. G. (2001). Universal fine structure of nematic hedgehogs. Journal of Physics A: Mathematical and General, 34, 829-838.

Kralj, S., Rosso, R., Virga, E. G. (2011). Curvature control of valence on nematic shells. Soft Matter 7, 670-683.

Mermin, N. D. (1976). The topological theory of defects in ordered media. Review of Modern Physics, 51, 591-648.

Nelson, D. R. (2002). Toward a tetravalent chemistry of colloids. Nano Letter, 2, 1125-1129. 
Samo KRALJ, Boris ABERŠEK, Irena KRALJ. Interplay between Art and Science in Education: "Music" Based Approach in Nanosciences

PROBLEMS

OF EDUCATION

IN THE $21^{\text {st }}$ CENTURY

Volume 61,2014

Rößler1, U. K., Bogdanov, A. N., Pfleiderer, C. (2006). Spontaneous skyrmion ground states in magnetic metals. Nature, 442, 797-801.

Skyrme, T. H. (1961). A non-linear field theory. Proceedings of the Royal Society, London Series A, 260, 127-138.

Vitelli, V., Turner, A. M. (2004). Anomalous coupling between topological defects and curvature. Physical Review Letters, 93, 215301, 1-4.

Vitelli, V., Nelson, D. R. (2006). Nematic textures in spherical shells. Physical Review, E 74, 021711, $1-18$.

Wright, D. C., Mermin, N. D. (1989). Crystalline liquids - the blue phases. Review of Modern Physics, $61,385-432$.

Zurek, W. H. (1976). Cosmological experiments in condensed matter. Physics Report, 276, 177-221.

Advised by Vincentas Lamanauskas, University of Šiauliai, Lithuania

Received: June 30, 2014

Accepted: August 28, 2014

\begin{tabular}{|ll|}
\hline Samo Kralj & $\begin{array}{l}\text { PhD., Professor, University of Maribor, Koro ka 160, } 2000 \text { Maribor, Slovenia. } \\
\text { E-mail: samo.kralj@uni-mb.si } \\
\text { Website: http://samo.kralj.fnm.uni-mb.sil }\end{array}$ \\
\hline Boris Aberšek & $\begin{array}{l}\text { PhD., Professor, University of Maribor, Koro ka 160, } 2000 \text { Maribor, Slovenia. } \\
\text { E-mail: boris.abersek@uni-mb.si }\end{array}$ \\
\hline Irena Kralj & $\begin{array}{l}\text { Teacher, High School in Celje (I.gimnazija v Celju), Kajuhova 2, 3000 Celje, Slovenia. } \\
\text { E-mail: neni.kralj@gmail.com }\end{array}$
\end{tabular}

